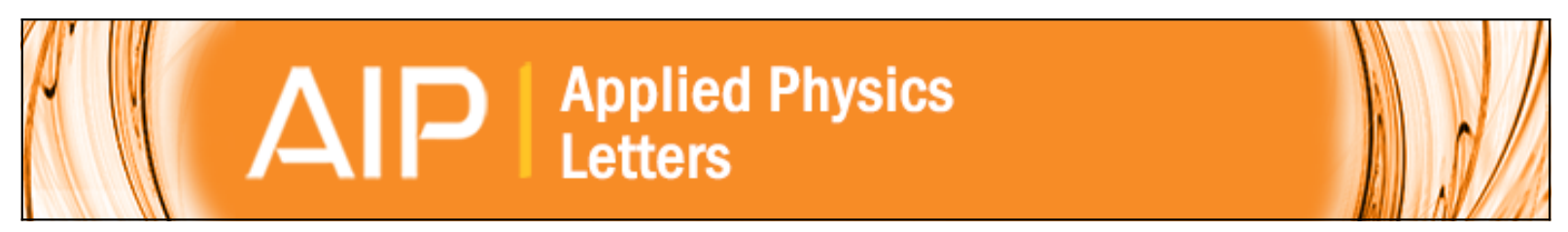

\title{
Thermal rectification in a fluid reservoir
}

Sohail Murad and Ishwar K. Puri

Citation: Applied Physics Letters 100, 121901 (2012); doi: 10.1063/1.3696022

View online: http://dx.doi.org/10.1063/1.3696022

View Table of Contents: http://scitation.aip.org/content/aip/journal/apl/100/12?ver=pdfcov

Published by the AIP Publishing

AlP Re-register for Table of Content Alerts 


\title{
Thermal rectification in a fluid reservoir
}

\author{
Sohail Murad ${ }^{1}$ and Ishwar K. Puri²,a) \\ ${ }^{1}$ Department of Chemical Engineering, University of Illinois at Chicago, Chicago, Illinois 60607, USA \\ ${ }^{2}$ Department of Engineering Science and Mechanics, Virginia Tech, Blacksburg, Virginia 24061, USA
}

(Received 23 January 2012; accepted 3 March 2012; published online 19 March 2012)

\begin{abstract}
An organized nonuniform mass distribution in solids leads to a monotonically varying thermal conductivity in a nanomaterial so that the heat flux is directionally dependent. We investigate through molecular dynamics simulations if the influence of an organized mass distribution in a fluid also leads to thermal rectification. Heat transfer is monitored in a water reservoir placed between two (hot and cold) silicon walls. The distribution of the fluid in the reservoirs is organized by applying an external force to each water molecule in a specified direction, creating a density gradient. This external force is smaller than the intermolecular forces in water, in most cases by much more than an order of magnitude. The simulations reveal that mass graded fluid-containing nanosystems can be engineered to possess an asymmetric axial thermal conductance that leads to greater heat flow in the direction of decreasing mass density. The rectification improves as the thermal conductivity is enhanced by increasing the fluid density adjacent to a hot wall, since doing so decreases the interfacial resistance and increases the heat flux. (C) 2012 American Institute of Physics. [http://dx.doi.org/10.1063/1.3696022]
\end{abstract}

Since mass disorder alters the thermal conductivity of a nanomaterial, ${ }^{1}$ an organized nonuniform mass distribution leads to a monotonically varying thermal conductivity $k$. In a nanoscale solid state device, this leads to greater heat flow in the direction of decreasing mass density, resulting in thermal rectification. $^{2}$ Topological asymmetry in a quasi onedimensional structure can also produce such a rectification. ${ }^{3}$ In all these cases, the conductivity $k$ and thus the heat flux $q^{\prime \prime}$ are directionally dependent. By switching the direction of the temperature gradient $(\Delta T) /(\Delta x)$ but keeping its overall magnitude constant will change $q^{\prime \prime}$, e.g., if done from left $(L)$ to right $(R)$ then $q_{L \rightarrow R}^{\prime \prime} \neq q_{R \rightarrow L}^{\prime \prime}$.

We hypothesize that a similarly structured nonuniform mass distribution in a fluid also leads to thermal rectification. We investigate the role of the solid-fluid interfacial (Kaptiza) resistance in facilitating rectification. In many cases, it is simpler to introduce surface modifications that exploit the interfacial hydrophobic — or hydrophilic — properties to engineer the interfacial heat transfer across solid-fluid interfaces. ${ }^{4-6}$ While there is an extensive literature on rectification in solid state systems, only a single entry discusses this phenomena across a solid-liquid (silica-water) interface. ${ }^{7}$ In the case of a silica-water interface, rectification occurs through the decrease in the hydrogen bonding in water at warmer temperatures that degrades the local bond network and the vibrational properties of water.

Our molecular dynamics simulations are designed to understand rectification when heat is transferred across a solid-fluid interface through a bulk fluid placed in a reservoir. The simulated system, shown in Fig. 1, consists of 1024 molecules each of water and silicon in a basic cyclically replicated parallelepiped. Two walls consisting of 512 $\mathrm{Si}$ atoms each (or $4 \times 4 \times 4$ unit cells) are placed at their normal equilibrium sites in a silicon crystal. These wall mole-

${ }^{a)}$ Electronic mail: ikpuri@vt.edu. cules are tethered to their equilibrium sites and allowed to vibrate using a simple harmonic potential. Initially, water molecules are uniformly distributed in the two reservoirs placed on either side of these walls. The axial ( $x$-wise) dimension of the system is much larger than the transverse $y$ - and $z$-directions to facilitate the simultaneous simulation of both bulk and molecular-scale characteristics. Thus, while the walls influence small portions of the fluid adjacent to them, ${ }^{4,5,8}$ the remaining fluid in the reservoirs exhibits bulk properties. The volumes of the reservoirs between the walls are equal.

All solid and fluid molecules are provided with initial Gaussian velocity distributions. We impart different average hot and cold temperatures $T_{h}$ and $T_{c}$ to the silicon walls using a Gaussian thermostat, which results in an average fluid temperature $T_{a v, f}=\left(T_{h}+T_{c}\right) / 2$ that is relatively invariant to the consequent heat flux. The $(N, V, T)$ simulations proceed with uniform step sizes of $1 \mathrm{fs}$ and are allowed to equilibrate to an energy minimum state. To maintain the desired temperatures, a Gaussian thermostat is applied separately on the entire hot and cold walls and on the fluid sections between them. Hence, the temperatures in these regions vary although the average temperature is constant. We report results for simulations that have progressed by $2 \times 10^{6}$ time steps to

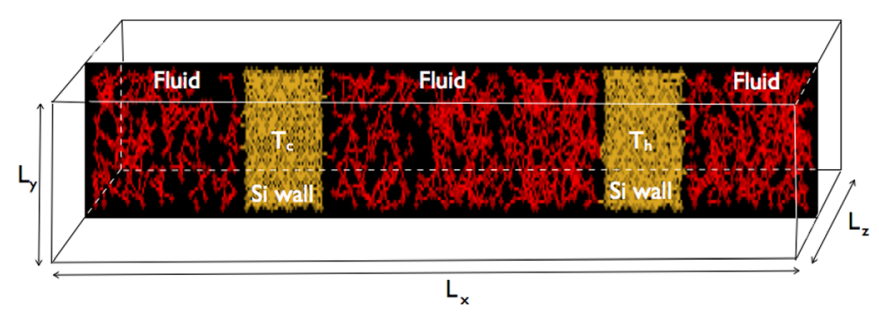

FIG. 1. (Color online) Schematic of the simulation domain, where $L_{x}=20.2$, and $L_{y}=L_{z}=2.04 \mathrm{~nm}$. The system is divided into 138 slabs along $L_{x}$, each with a volume of $6.09 \times 10^{-28} \mathrm{~m}^{3}$. The Si walls include slabs $30-43$ and $99-113$. 
$2 \mathrm{~ns}$ at which time the systems have reached their steady states. Longer simulations with $4 \times 10^{6}$ steps confirm that no further changes occur in the temperature or density profiles.

We place the water between a $403 \mathrm{~K}$ cold wall and a $1209 \mathrm{~K}$ hot wall so that the globally averaged system temperature is $806 \mathrm{~K}$ and an average water density $\rho=487.5 \mathrm{~kg} / \mathrm{m}^{3}$. The bulk fluid is on average supercritical under these conditions. The relatively high wall temperatures facilitate larger heat transfer rates that minimize data scatter but do not otherwise alter the fundamental aspects of rectification. Indeed, we repeated most of the simulated cases with the wall and system temperatures significantly reduced. These led to bulk liquid water in the reservoirs and provided similar results to those reported but required much longer simulations involving $8 \times 10^{6}$ time steps or longer and produced greater scatter in the resulting data.

The molecular dynamics algorithm uses the quaternion method with a fifth-order Gear predictor-corrector algorithm for translational motion and a fourth-order predictorcorrector algorithm for rotational motion. ${ }^{9,10}$ Intermolecular interactions are described by the potential model $u_{i j}=$ $4 \varepsilon_{i j}\left(\left(\sigma_{i j} / r_{i j}\right)^{12}-\left(\sigma_{i j} / r_{i j}\right)^{6}\right)+\left(q_{i} q_{j}\right) / r_{i j}$, where $\sigma_{\mathrm{ij}}$ and $\varepsilon_{\mathrm{ij}}$ denote the L-J interaction parameters, $r_{i j}$ is the scalar distance between sites $i$ and $j$, and $q_{i}$ and $q_{j}$ are the charges on these sites (since water contains a partial $+v e$ charge for $\mathrm{H}$ and $-v e$ for $\mathrm{O}$ ). For water, we use the simple point charge (SPC) potential which is known to represent water properties realistically. ${ }^{9}$ While we do not use the more accurate Stillinger-Weber potential model for Si that employs threebody interactions to enforce the correct coordinated tetrahedral bonded structure of $\mathrm{Si}^{11}{ }^{11}$ we are able to nevertheless enforce this arrangement by tethering the $\mathrm{Si}$ atoms to their equilibrium sites. Hence, the simpler L-J model is an acceptable alternative here. Lorentz-Berthelot mixing rules are used to model cross interactions and the reaction field method for long-range interactions ${ }^{12,13}$ with parameters corresponding to the average state conditions of the systems and a potential cutoff of $9.5 \AA$. Although the reaction field method is intended for homogeneous systems, it also works well for nonhomogeneous systems, e.g., the more accurate particle-particle mesh method provides results comparable with the reaction field method. ${ }^{14}$ Use of the larger cutoff distance further justifies our methodology. Our previous studies, which also involved nonhomogeneous systems, showed that long range interactions do not seem to significantly contribute to transport processes in the type of system investigated here. $^{15,16}$

Since our hypothesis requires the organization of a fluid mass distribution in the reservoirs, an external force $F$ is added to each water molecule in the $x$-direction, thus creating a density gradient in the system. For consistency and to maintain the symmetry of our periodic simulation systems, the forces in the central section between the two Si walls, and those imposed on either sides of these two walls are applied in opposite directions. These external forces are varied between 0 and $3 \mathrm{pN}$. In comparison, the intermolecular forces in water are larger than these forces by more than an order of magnitude. While the imposed force is simply added to the intermolecular force experienced by each water molecule in the simulations, it should be possible to include such directional forces in fluid systems by employing electric and magnetic fields.

In addition to Case I that pertains to an otherwise undisturbed system that has no imposed force on its fluid molecules, we consider six pairs of other cases, each denoted as (a) and (b), as reported in Table I. The system is divided in the $x$-direction into 138 slabs, each of $6.09 \times 10^{-28} \mathrm{~m}^{3}$ volume, in which the local properties are averaged. Figures 2(a) and 2(b) present the system response for Case I and Cases $\mathrm{II}(\mathrm{a})$ and II(b). The centers of the walls contain a larger number of atoms due to the atomic arrangements of their constituent $\mathrm{Si}$ crystals, as shown in Fig. 2(a). The observed peaks in the silicon density profiles result from the temperature variations within the silicon wall and the resulting thermal fluctuations. These peaks will not be observed in constant temperature systems. The water density for Case I in the vicinity of the cold wall is slightly higher than the corresponding value adjacent to the warmer wall due to a local temperature effect. The temperature profile across the solidfluid interfaces reveals a characteristic temperature discontinuity ${ }^{17} \Delta T$ due to phonon scattering ${ }^{18-20}$ that impedes phonon transport through the thermal (or Kapitza) resistance ${ }^{21}$ $R=\Delta T / q^{\prime \prime}\left(\mathrm{m}^{2} \mathrm{~K} / \mathrm{W}\right){ }^{4,5,8}$ This resistance is modeled classically in our studies by considering the thermal vibrations of the $\mathrm{Si}$ atoms in the silicon crystals.

An applied force increases the relative numbers of fluid molecules $N_{h}$ that lie adjacent to the hot wall, thus producing a higher mass gradient $\mathrm{d} N / d x$ against the direction of heat transfer. As shown in Fig. 2(b), when there is a nonuniform water mass distribution, the thermal behavior is quite different from the results of Fig. 2(a). The heat flux is enhanced for larger $N_{h}$ near the hot wall both because of the variations in the water thermal conductivity between the two walls, and the decrease in the interfacial, or Kapitza resistance. For -ve $F, \mathrm{~d} N / d x$ assumes a $+v e$ value along the direction of heat transfer since the fluid mass is now graded from the cold to the hot wall, i.e., there are more cold fluid molecules than there are warmer ones. In this case, $q^{\prime \prime}$ is diminished. Thus our simulations clearly demonstrate that, in accord with previous results for a solid state device, mass graded fluid-containing systems can also be engineered to possess an asymmetric axial thermal conductance that leads to

TABLE I. Dimensionless force $F=(\sigma / \varepsilon)$ applied to each water molecule for the 13 simulated cases, where $\varepsilon / k_{B}=806 \mathrm{~K}$ and $\sigma=0.19 \mathrm{~nm}$ in our simulations. With these reducing factors a unit of force equals $58.57 \mathrm{pN}$, i.e., $F=0.05$ is equivalent to 2.93 pN. Case I represents the base simulation with $F=0$. For the other six case pairs, the forces are applied either (a) towards (+ve F), or (b) away from ( $-v e F$ ) the hot $\mathrm{Si}$ wall.

\begin{tabular}{lccccc}
\hline \hline Cases & I & II (a), (b) & III (a), (b) & IV (a), (b) & V (a), (b) \\
\hline$F(a),(b)$ & N/A & \pm 0.05 & \pm 0.0375 & \pm 0.025 & \pm 0.0125 \\
\hline \hline
\end{tabular}



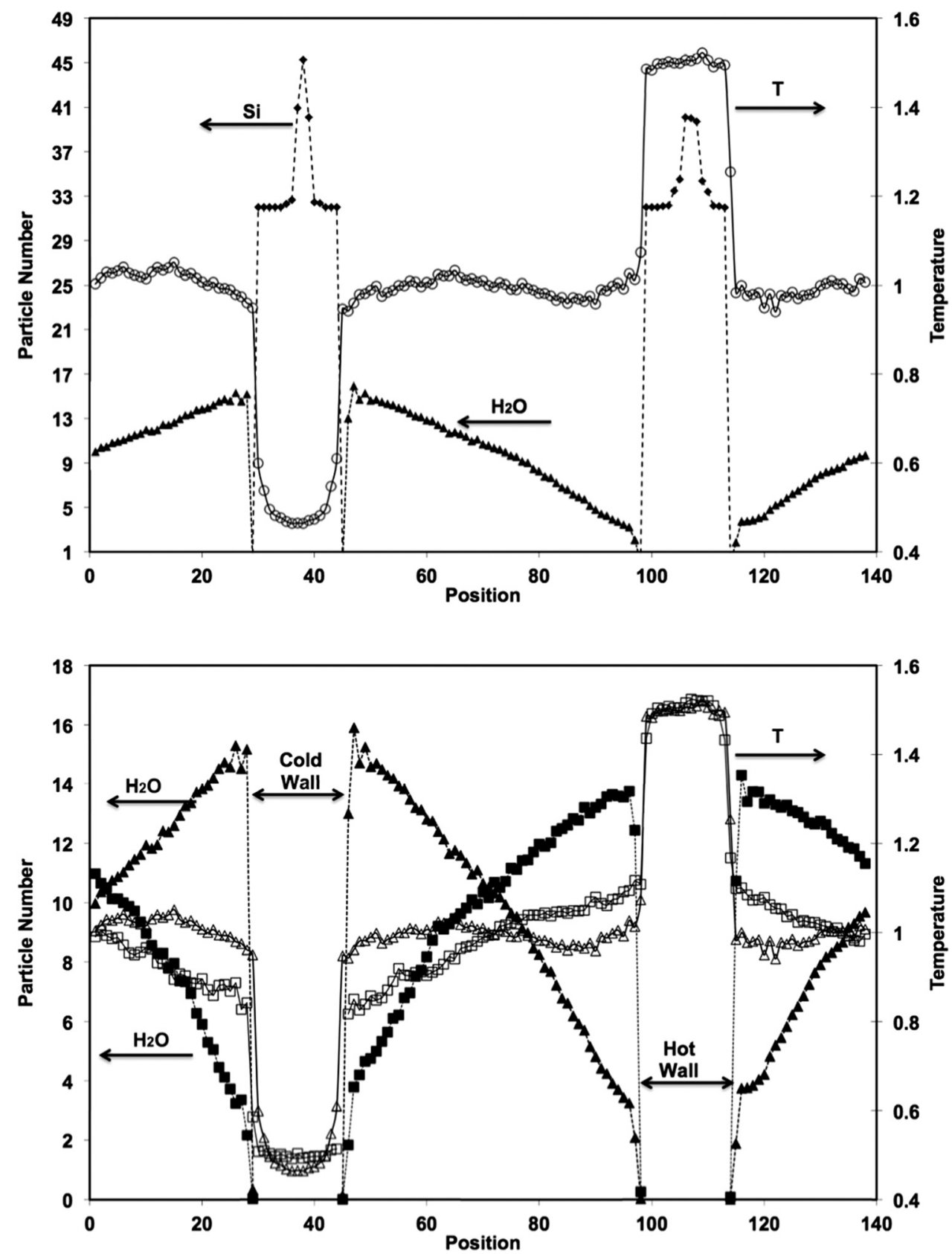

FIG. 2. The behavior of the (a) base undisturbed system of Case I (with symbols $\bullet$ : number of $\mathrm{Si}$ atoms, $\boldsymbol{\Delta}$ : number of $\mathrm{H}_{2} \mathrm{O}$ molecules, and $\bigcirc$ : temperature), and (b) Cases II (a) and (b) with $F= \pm 0.05$ (where for $+v e F, \boldsymbol{\square}$ : number of $\mathrm{H}_{2} \mathrm{O}$ molecules and $\square$ : temperature, and for $-v e F, \boldsymbol{\Delta}$ : number of $\mathrm{H}_{2} \mathrm{O}$ molecules and $\Delta$ : temperature). The dimensionless temperatures should be multiplied by $806 \mathrm{~K}$ to recover the units of $\mathrm{K}$. The system dimension in the $x$-direction is $20.2 \mathrm{~nm}$, which is divided into 138 slabs in each of which the local properties are averaged. The position numbers of these slabs are shown, where each position number corresponds to an increment of $1.464 \AA$. Recall that a unit of force equals $58.57 \mathrm{pN}$ so that $F= \pm 0.05$ is equivalent to $\pm 2.93 \mathrm{pN}$. greater heat flow in the direction of decreasing mass density. $^{2}$

As noted, increasing values of $F$ (and thus $N_{h}$ ) lead to a decrease in the interfacial resistance $R$, which lowers the interfacial solid-fluid temperature difference $\Delta T$. Hence, Figure 2(b) also shows that $\Delta T$ decreases at the hot interface when $N_{h}$ increases. The imposition of $F=0.05$, which significantly increases the fluid density adjacent to the hot wall but decreases it near the cold wall, decreases $\Delta T$ across both the hot and cold solid-fluid interfaces as compared to Case I shown in Fig. 2(a) for $F=0$. The decrease in $\Delta T$ near the cold surface is counterintuitive. Since the fluid temperature and density near the cold surface are both now lower, the interfacial resistance should be larger ${ }^{4,5,8}$ as should $\Delta T$.

We attribute the lower interfacial across the cold wall to water condensation, which increases the fluid density adjacent to the solid wall just as a hydrophilic surface does. ${ }^{8}$ The structural ordering of a fluid at a solid-fluid interface enhances the local conductivity, reducing the interfacial resistance, which in turn decreases $\Delta T^{4,22}$ The critical temperature of water is $647 \mathrm{~K}$. For $F=0.05$, the fluid temperature near the wall is $\sim 645 \mathrm{~K}$, whereas the wall surface temperature is $\sim 410 \mathrm{~K}$. When $F=0$, the fluid temperature is $\sim 725 \mathrm{~K}$ and the wall surface temperature is $\sim 435 \mathrm{~K}$. Since the system pressure is above the critical pressure, we note that when $F=0$, the fluid adjacent to the wall is supercritical. For $F=0.05$, the fluid exists as liquid there, which leads to the lower value of $R$ observed due to water condensation.

When $F=-0.05$, the external force increases the density near cold wall but decreases $N_{h}$ adjacent to the hot wall. In this case, the dimensionless bulk fluid temperature simply oscillates around its average value of unity, i.e., there is negligible heat transfer through the fluid reservoir despite the significant temperature difference between its two enclosing walls. This unusual situation is the result of the very significant Kapitza resistances across both hot and cold interfaces that prevent heat transfer. Figure 2(b) shows that, unlike when $F=0$ and 0.05 , almost all of the temperature decrease 


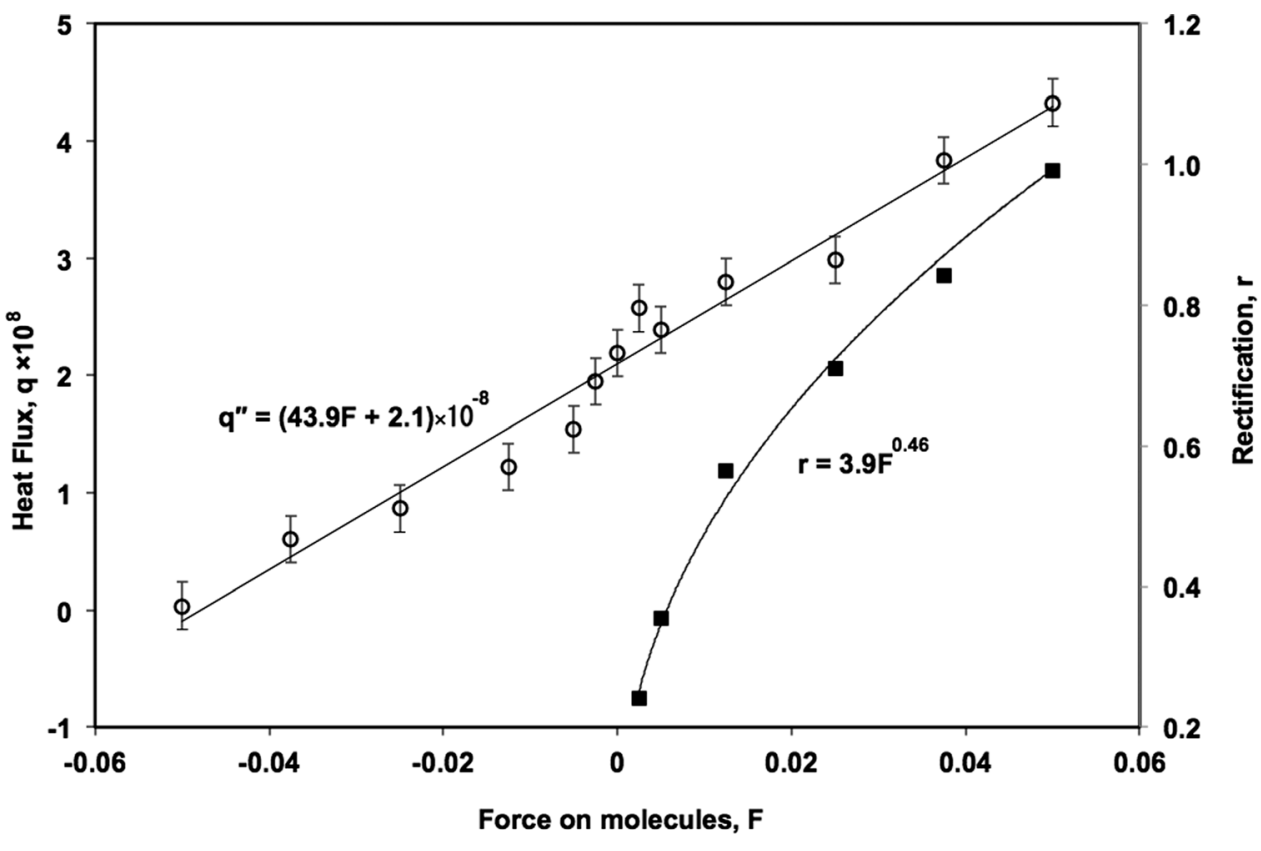

FIG. 3. The variation in the heat flux $(\mathrm{O}$, in units of $\mathrm{W})$ and the thermal rectification (ם) with respect to the applied force $F$ that creates a mass gradient in the fluid reservoir. A unit of force equals $58.57 \mathrm{pN}$ so that, for instance, $F=0.05$ is equivalent to $2.93 \mathrm{pN}$. Larger $F$ values increase the numbers of fluid molecules $N_{h}$ that lie adjacent to the hot wall and thus produce a higher mass gradient $\mathrm{d} N / d x$ along the direction of heat transfer. Smaller values diminish $N_{h}$ and hence the magnitude of $\mathrm{d} N / d x$. For -ve $F, \mathrm{~d} N / d x$ is positive against the direction of heat transfer since the fluid mass is now graded from the cold to the hot wall, i.e., there are more cold fluid molecules than warmer ones. The heat flux $q \approx(43.9 F+2.1) \times 10^{-8} \mathrm{~W}$, and the rectification $r \approx 3.9 F^{0.5}$. across the fluid occurs in its interfacial layers when $F=-0.05$. When $F$ is nonnegative, the temperature gradients are distributed between the interfacial layers and the bulk fluid, illustrating the smaller relative resistance to heat transfer in these regions. Another consequence of higher $q^{\prime \prime}$ is a larger effective thermal conductance across the solidfluid interface. This conductance increase leads to a relative smoothening of temperature gradients within the cold wall.

Figure 3 shows the variation in the heat flux with respect to $F$. The flux $q$ ( $=q^{\prime \prime} A$, with $A$ denoting the cross sectional area) follows the linear relation $q \approx(43.9 F+2.1) \times 10^{-8} \mathrm{~W}$. If $\mathrm{d} N / \mathrm{d} x$ assumes a large enough value against the direction of heat transfer, $q$ can become negligible. One strategy to impose thermal rectification would be to make the hot wall hydrophilic and the cold wall hydrophobic. This corresponds to the case of $+v e F$ in Figs. 2(b) and 3, where $N_{h}$ and there- fore $q$ are enhanced. Switching the surface properties of the hot and cold walls would be analogous to applying -ve $F$ and decreasing $q$. To determine the influence that switching the direction of $F$ has on $q$, we define thermal rectification for any pair of the six cases listed in Table $\mathrm{I}$ as $r=\left(q_{+F}-q_{-F}\right) / q_{+F}$. Figure 3 reveals the relationship $r \approx 3.9 F^{\alpha}$, where $\alpha \approx 0.5$. This shows that small initial applications of $F$ are very effective in increasing $r$.

Increasing $F$ also increases $N_{h}$ and thus the density $\rho$ adjacent to the hot wall. The conductivity of a fluid increases with density. ${ }^{23,24}$ Hence, the thermal rectification results from the thermal conductivity enhancement due to increasing $N_{h}$ that decreases the interfacial resistance $R$, increasing $q$. This is corroborated by Fig. 4 that shows that $r \approx 0.15 N_{h}-1$ (where $N_{h}$ is taken for $+v e F$ values). The decrease in $R$ as $N_{h}$ increases is shown in Fig. 4, which is

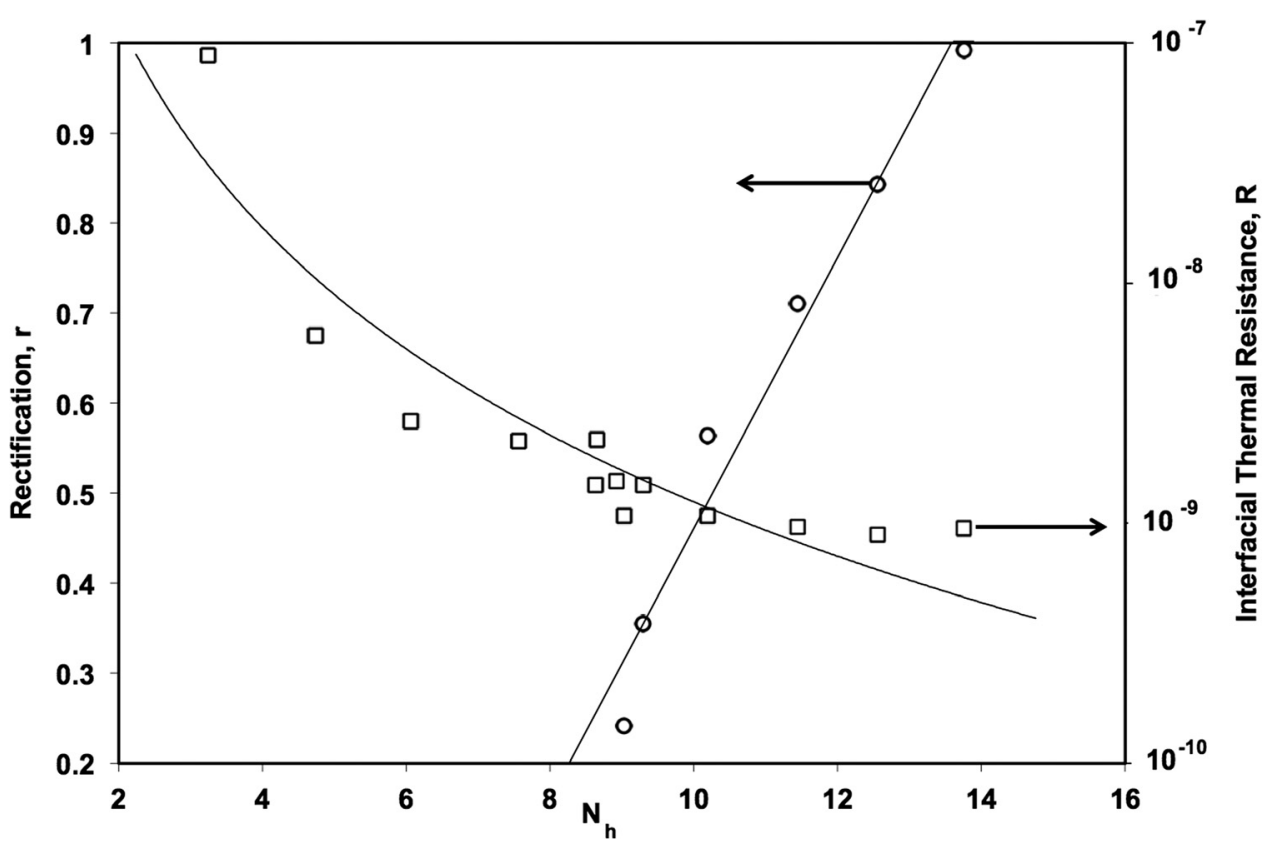

FIG. 4. The variation in the rectification $r$ with the number of water molecules $N_{h}$ adjacent to the hot wall (corresponding to the values for $+v e F$ ) for the six pairs of cases when $F$ is nonzero. On the secondary axis, the variation of the interfacial thermal resistance $R$ (in units of $\mathrm{m}^{2} \mathrm{~K} \mathrm{~W}^{-1}$ ) with $N_{h}$ is presented for all the cases listed in Table I. Multiplying $N_{h}$ (which changes as the applied force $F$ is varied) by $49.1 \mathrm{~kg} \mathrm{~m}^{-3}$ molecule $^{-1}$ returns the fluid density in the slab adjacent to the hot wall. The figure reveals the relations $r \approx 0.15 N_{h}-1$ (where $N_{h}$ is taken for +ve $F$ values), and $R \propto \rho^{-2.8}$. 
expected from both the acoustic and diffuse mismatch models of phonon transport. ${ }^{21}$ The results presented in Fig. 4 for these particular simulations show that $R \propto \rho^{-2.8}$.

In summary, we show that thermal rectification is possible in mass graded liquid systems to produce a greater heat flux in the direction of decreasing mass density but a lower flux in the direction of increasing density. As for a solid material, this effect in a fluid is also based on thermal conductivity modifications due to changes in the mass density. This density difference has a significant influence on the solidfluid interfacial resistance. The density variations that we have imposed can be accomplished in practice by employing electric and magnetic fields. Another strategy would be to make the hot surface hydrophilic and the other cold surface hydrophobic, which is similar to imposing $+v e F$.

${ }^{1}$ G. Balasubramanian, I. K. Puri, M. C. Bohm, and F. Leroy, Nanoscale 3(9), 3714 (2011).

${ }^{2}$ C. W. Chang, D. Okawa, A. Majumdar, and A. Zettl, Science 314(5802), 1121 (2006).

${ }^{3}$ J. Hu, X. Ruan, and Y. P. Chen, Nano Lett. 9(7), 2730 (2009).

${ }^{4}$ S. Murad and I. K. Puri, Appl. Phys. Lett. 92(13), 133105 (2008).
${ }^{5}$ S. Murad and I. K. Puri, Chem. Phys. Lett. 476(4-6), 267 (2009).

${ }^{6}$ P. A. E. Schoen, B. Michel, A. Curioni, and D. Poulikakos, Chem. Phys. Lett. 476(4-6), 271 (2009).

${ }^{7}$ M. Hu, J. V. Goicochea, B. Michel, and D. Poulikakos, Appl. Phys. Lett. 95(15), 151903 (2009).

${ }^{8}$ S. Murad and I. K. Puri, Chem. Phys. Lett. 467(1-3), 110 (2008).

${ }^{9}$ J. Chandrasekhar, S. F. Smith, and W. L. Jorgensen, J. Am. Chem. Soc. 106(10), 3049 (1984).

${ }^{10}$ D. J. Evans and S. Murad, Mol. Phys. 34(2), 327 (1977).

${ }^{11}$ F. H. Stillinger and T. A. Weber, Phys. Rev. B 31(8), 5262 (1985).

${ }^{12}$ I. G. Tironi, R. Sperb, P. E. Smith, and W. F. Vangunsteren, J. Chem. Phys. 102(13), 5451 (1995).

${ }^{13}$ H. J. C. Berendson, J. Postma, and W. F. van Gunsteren, in Intermolecular Forces, edited by B. Pullman (Reidel, Dordecht, 1981).

${ }^{14}$ R. Gargallo, P. H. Hunenberger, F. X. Aviles, and B. Oliva, Protein Sci. 12(10), 2161 (2003).

${ }^{15}$ S. Murad and J. Lin, Chem. Eng. J. 74(1-2), 99 (1999).

${ }^{16}$ R. O. Watts, Mol. Phys. 28(4), 1069 (1974).

${ }^{17}$ P. L. Kapitza, J. Phys. (USSR) 4, 181 (1941).

${ }^{18}$ L. J. Challis, J. Phys. C 7(3), 481 (1974).

${ }^{19}$ J. L. Barrat and F. Chiaruttini, Mol. Phys. 101(11), 1605 (2003).

${ }^{20}$ C. Oligschleger and J. C. Schon, Phys. Rev. B 59(6), 4125 (1999).

${ }^{21}$ G. L. Pollack, Rev. Mod. Phys. 41(1), 48 (1969).

${ }^{22}$ G. Balasubramanian, S. Banerjee, and I. K. Puri, J. Appl. Phys. 104(6), 064306 (2008).

${ }^{23}$ J. F. D. Smith, Ind. Eng. Chem. 22(11), 1246 (1930).

${ }^{24}$ H. F. Weber, Wied. Ann. 10, 304 (1880). 